\title{
Morphological alterations in peripheral blood smears of covid 19 patients: study of two cases from district hopsital, pali
}

\author{
H R Bohra, A. Tandon \\ Corresponding Author: \\ H R Bohra \\ Assistant Professor Pathology, \\ Government Medical College (RAJMES) \\ Pali Rajasthan, \\ Email: dr.harishbohra@gmail.com
}

\section{Introduction:}

Corona virus disease 2019 (COVID-19) caused by Novel Corona Virus (2019-nCOV), is attracting the attention of the whole world from December 2019 with wide range of clinical manifestations extending from a mild flu-like illness to life-threatening forms (1). Globally, till 11th June 2021, there have been 174,502,686 confirmed cases of COVID-19, including $3,770,361$ deaths, reported to WHO. Universal implementation of corona etiquettes, vaccination, extensive diagnosis of COVID 19 by RTPCR and enhancement of prognostic and therapeutic facilities decreases the new cases over 3 million and over 73000 new deaths (2). Despite differences among countries, the overall in-hospital mortality is high, being reported up to $30 \%$ [3]. The reasons underlying such a different clinical behavior are still unknown; thus, it is difficult to predict who will develop a severe clinical picture and who, conversely, will show a mild disease. Blood cell count is a simple, highly informative, and universally available laboratory test. Furthermore, preliminary reports suggest that blood cell count is valuable tool for clinical risk stratification [4 - 6].

Circulating biomarkers that can represent inflammation and immune status are potential predictors for the prognosis of COVID-19 induced systemic inflammatory responses in patients [7]. Peripheral white blood cell (WBC) count, neutrophil (NEU)-to-lymphocyte (LYM) ratio i.e absolute neutrophils count divided by absolute lymphocytes count (NLR), derived NLR ratio (dNLR, neutrophils count divided by the result of WBC count minus neutrophil count), platelet-tolymphocyte ratio i.e absolute platelets count divided by absolute lymphocytes count (PLR) and lymphocyte-to-monocyte ratio i.e. absolute lymphocytes count divided by absolute monocytes count (LMR) widely investigated as useful diagnostic and prognostic markers (8). Very few studies show morphological changes in White Blood cell series in peripheral smears can be helpful in early diagnosis and even prognosis of COVID -19 disease (9-13). We are 
presenting the morphological changes in WBCs series in peripheral smear of two COVID 19 patients from government district hospital Pali Rajasthan.

\section{Case 1}

A 55-year old previously healthy woman was admitted with fever and cough. Chest X-ray and CT showed features of viral pneumonitis. Her nasopharyngeal swab was positive for severe acute respiratory distress syndrome coronavirus-2 (SARS-CoV-2) by reverse transcription (RT)-PCR. Her complete blood count (CBC) showed leucocytosis (Total Leukocyte Count =17,000/ul) with neutrophilia, relative lymphocytopaenia, monocytopaenia and $\mathrm{N}$ : L ratio was 8.2. Her detailed analysis of the peripheral blood film (PBF) time of admission revealed peculiar findings. Neutrophils showed heavily clumped chromatin dense basophilic toxic granules and cytoplasmic vacuoles. C-shaped, fetus-like nuclei were noted with aberrant nuclear projections, which we named as COVID nuclei (figure 1).

\section{Case 2}

Another 35 years old patient came to OPD with history of fever and cough since 4 days. His nasopharyngeal swab was positive for severe acute respiratory distress syndrome coronavirus-2 (SARS-CoV-2) by reverse transcription (RT)-PCR. His complete blood count showed TLC = $8000 /$ ul but N/L ratio was 3.5 and other CBC parameters are within normal limits. His peripheral smear showed most of the lymphocytes showed round to indented nuclei, condensed chromatin, along with abundant pale blue cytoplasm with cytoplasmic pod formation and apoptosis were also observed in a few lymphocytes (Fig 2).

\section{Discussion}

Yang et al found age, NLR, d-NLR, CRP, LMR and PLR were potential diagnostic marker to discern non severe and severe Covid 19 infection with values of Area under Curve (AUC) analysis were $0.743,0.841,0.815,0.714,0.265$, and 0.784 respectively. No unified laboratory reference value was found. The optimal cut-off values were 3.3, 2.8, and 180 for NLR, d-NLR, and PLR, respectively. The highest specificity and sensitivity were 0.636 and $0.88,0.55$ and 0.84, 0.44 and 0.77 for NLR, d-NLR, and PLR, respectively. Evaluated NLR (HR 2.46, 95\% CI 1.98-4.569), d-NLR (HR 1.92, 95\% CI 0.817-3.496), and PLR (HR 1.023, 95\% CI 0.921-1.756) were considered independent factors associated with COVID-19 progression. Other various studies also proved N: L ratio is an independent prognostic marker in COVID 19 infection (4-6). In our both cases we find increased $\mathrm{N}$ : L ratio but we could not evaluate its prognostic role as patients lost to follow up.

Alteration in morphology of cells of the peripheral blood in COVID-19 as is evident by few studies [9-13]. In a study of 28 patients with confirmed COVID-19 infection with varying degrees of severity, morphological and functional changes of monocytes/ macrophages were noted using wright stained smears and flow cytometry, that was predictive of the severity of 
disease, likelihood of ICU admission, length of hospital stay and full recovery [9] Singh et al in his case report of COVID 19 patient, found following changes in peripheral blood smear.

Neutrophils showed heavily clumped chromatin with toxic granules and cytoplasmic vacuoles. Nuclear detachment with elongated nucleoplasm and ring-shaped nuclei were seen with platelet surface attachment. C-shaped, fetus-like nuclei were noted with aberrant nuclear projections they named as COVID nuclei. Most of the lymphocytes were seen as large granular lymphocytes (LGL) with round to indented nuclei, condensed chromatin, prominent nucleoli in a few, along with abundant pale blue cytoplasm with distinct variably sized azurophilic granules. Natural killer cell like lymphocytes with cytoplasmic pod formation and apoptosis were also observed in a few lymphocytes. These might represent natural killer cells or cytotoxic $\mathrm{T}$ lymphocytes. Activated monocytes were seen which showed marked anisocytosis with prominent cytoplasmic vacuolization and few granules. Nuclei were large, having fine chromatin with nuclear blebbing in a few. Nuclear overlapping by vacuoles was observed in some cells, Platelets were adequate, with a few giant forms and focal platelet attachment on the surface of all forms of leukocytes.

Nath et al studied pathomorphological changes in peripheral blood smear of 26 COVID 19 patients with the NLR and LMR derived from the CBC and with the clinical and molecular status (RT-PCR) of the patient at the time of admission and during the hospital stay (13). He found predominantly Normocytic normochromic picture $(69.2 \%, 18 / 26)$, followed by dimorphic (microcytic-macrocytic) $(15.4 \%, 4 / 26)$ Microcytic hypochromic $(7.7 \%, 2 / 26)$ and macrocytic $(7.7 \%, 2 / 26)$ in both the first sample and on follow up sample. Polymorphs having dyspoietic

features like hypolobation, ring shaped polymorph nucleus, cytoplasmic vacuoles, coarse granules mimicking toxic granules and some hypogranular polymorphs Apoptotic cells reminiscent of polymorphs with nuclear fragmentation were seen. Atypical lymphoid cells, large granular lymphocytes and monocytoid and plasmacytoid lymphoid cells were seen routinely. In our report both patients have normocytic normochromic picture; neutrophils with basophilic granules and $\mathrm{C}$ shaped ring nuclei and activated monocytoid lymphocytes at the time of admission only. He found morphological changes polymorphs in COVID-19 cases despite a normal NLR (CBC parameter). 20 cases despite a normal LMR (LMR>2.1) there were changes in lymphocyte and monocyte morphology present in peripheral smear (significant LMR<2.1). Explaining crucial role of peripheral smear examination decide COVID 19 infection before RT PCR result (13). We found changes in neutrophils and lymphocytes as described above.

\section{Conclusion:}

Our goal is to describe the peculiar morphological findings of affected leucocytes, which would help physicians suspect a diagnosis in the absence of a negative RT-PCR or antibody results. If confirmed in larger studies, these morphological features along with blood count would be helpful in the screening, diagnosis and management of these patients at all levels of healthcare. 


\section{Limitations:}

We could not perform follow up study and we had very less patient's data.

(Disclosure: Co-author (AT) help in concept and editing. The cases belong to first and corresponding author (HV).)

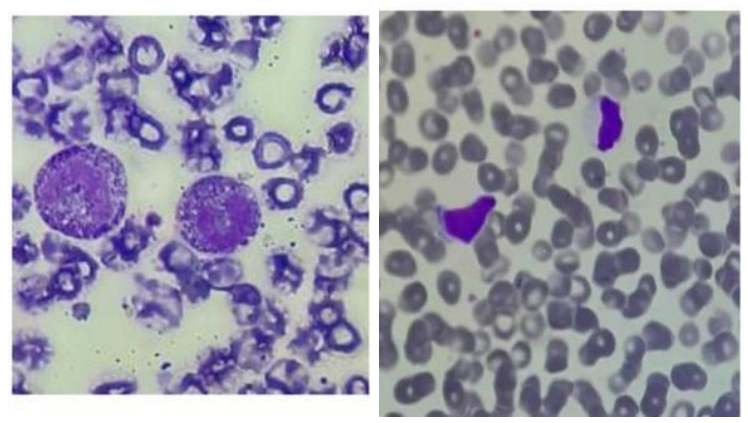

Figure 1 (a) neutrophils and (b) activated lymphocyte in COVID-19

\section{References:}

1. Q. Li, X. Guan, P. Wu, X. Wang, L. Zhou, Y. Tong, R. Ren, K.S.M. Leung, E.H.Y. Lau, J.Y. Wong, et al., Early transmission dynamics in Wuhan, China, of novel coronavirus- infected pneumonia, N. Engl. J. Med. (2020) 10-1056.

2.https://www.who.int/publications/m/item/weekly-epidemiological-update-on-covid-19---1june-2021

3. A. B. Docherty, E. M. Harrison, C. A. Green et al., "Features of 20133 UK patients in hospital with covid-19 using the ISARIC WHO clinical characterization protocol: prospective observational cohort study," BMJ, vol. 369, article m1985, 2020.

4. Liu, J., Liu, Y., Xiang, P. et al. Neutrophil-to-lymphocyte ratio predicts critical illness patients with 2019 coronavirus disease in the early stage. J Transl Med .2020; 18:206

5. Lissoni P, Rovelli F, Monzon A, Privitera C, Messina G, Porro G. Evidence of Abnormally Low Lymphocyte-To-Monocyte Ratio In COVID-19-Induced Severe Acute Respiratory Syndrome. J Immuno Allerg. 2020; 1(2):1-6.

6. Ai-Ping Yanga, Jian-ping Liub,*, Wen-qiang Taoc, Hui-ming Lib; The diagnostic and predictive role of NLR, d-NLR and PLR in COVID-19 patients, International Immunopharmacology 84 (2020) 106504.

7. N. Zhu, D. Zhang, W. Wang, X. Li, B. Yang, J. Song, X. Zhao, B. Huang, W. Shi, R. Lu, et al., A Novel Coronavirus from Patients with Pneumonia in China, 2019, N. Engl. J. Med. (2020) $10-1056$. 
8. N. Xiang, F. Havers, T. Chen, Y. Song, W. Tu, L. Li, Y. Cao, B. Liu, L. Zhou, L. Meng, et al., Use of national pneumonia surveillance to describe influenza A(H7N9) virus epidemiology, China, 2004-2013, Emerg. Infect. Dis. 19 (11) (2013) 1784-1790.

9. Zhang D, Guo R, Lei L, Liu H, Wang Y, Wang Y, et al. COVID-19 infection induces readily detectable morphological and inflammation-related phenotypic changes in peripheral blood monocytes, the severity of which correlate with patient outcome. medRxiv. 2020

10. Zini G, Bellesi S, Ramundo F, d'Onofrio G. Morphological anomalies of circulating blood cells in COVID-19. Am J Hematol. 2020;95: 870-872

11. A Singh, N Sood, V Narang A Goyal; Morphology of COVID-19-affected cells in peripheral blood film, BMJ Case Rep 2020;13:e236117.

12. Singh, S., Madan, J., Nath, D., \& Tiwari, N; Peripheral Blood Smear Morphology- A Red Flag in COVID-19. IJTDH; 2020; 41(8), 54-58.

13. Nath D, Madan U, Singh S, Tiwari N, Mdadan J, Agrawal R; CBC parameters and morphological alterations in peripheral blood cells in COVID-19 patients: Their significance and correlation with clinical course, International Journal of Health and Clinical Research, 2020;3(10):95-108.

How to Cite this article As: H R Bohra \& A. Tandon (2021). Morphological alterations in peripheral blood smears of covid 19 patients: study of two cases from district hopsital, pali. National Medicos Organisation Journal (एन एम ओ जर्नल) (ISSN-2348-3806), 15 (1), 14-18. Retrieved from https://nmojournal.org/index.php/nmojournal/index 